\title{
Four-loop master integrals and hypergeometric functions
}

\section{Stefano Laporta*}

Dipartimento di Fisica e Astronomia, Università di Padova, Via Marzolo 8, I-35131 Padova, Italy

Istituto Nazionale Fisica Nucleare, Sezione di Padova, Via Marzolo 8, I-35131 Padova, Italy

E-mail: stefano.laporta@pd.infn.it

We recall the analytical result of the 4-loop QED contribution to the electron $g$-2 and slope, which contain elliptic constants. We describe the relations between four elliptic constants which have ${ }_{4} F_{3}$ hypergeometric expressions. In particular we consider a quadratic relation found by Broadhurst and Mellit, and, by introducing a free parameter, we generalize it to a new quadratic relation between values of the hypergeometric ${ }_{4} F_{3}$.

MathemAmplitudes 2019: Intersection Theory \& Feynman Integrals 18-20 December 2019

Padova, Italy

${ }^{*}$ Speaker. 


\section{QED contributions}

In QED the anomalous magnetic moment, and the slope of the Dirac form factor can be expanded perturbatively in powers of $(\alpha / \pi)$

$$
\begin{aligned}
(g-2) / 2=F_{2}(0) & =\mathscr{C}_{1}(\alpha / \pi)+\mathscr{C}_{2}(\alpha / \pi)^{2}+\mathscr{C}_{3}(\alpha / \pi)^{3}+\mathscr{C}_{4}(\alpha / \pi)^{4}+\ldots, \\
m^{2} F_{1}^{\prime}(0) & =\mathscr{A}_{1}(\alpha / \pi)+\mathscr{A}_{2}(\alpha / \pi)^{2}+\mathscr{A}_{3}(\alpha / \pi)^{3}+\mathscr{A}_{4}(\alpha / \pi)^{4}+\ldots
\end{aligned}
$$

The coefficients $\mathscr{C}_{i}$ and $\mathscr{A}_{i}$ are pure numbers and can be extracted from the Feynman diagrams of the theory as linear combination of (a large number of) Feynman integrals. These combinations are to be reduced to a linear combination of (irreducible) master integrals by solving of large systems of IBP identities.

The contributions from one, two and three loops are known in analytical form since a long time [1-5] [6-8]:

$$
\begin{aligned}
\mathscr{C}_{1}= & \frac{1}{2}, \\
\mathscr{A}_{1}= & -\frac{1}{8}-\frac{1}{3} \ln \frac{\Delta E}{m}+\frac{5}{18}, \\
\mathscr{C}_{2}= & \frac{197}{144}+\frac{1}{12} \pi^{2}-\frac{1}{2} \pi^{2} \ln 2+\frac{3}{4} \zeta(3)=-0.328478965 \ldots, \\
\mathscr{A}_{2}= & -\frac{4819}{5184}-\frac{49}{432} \pi^{2}+\frac{1}{2} \pi^{2} \ln 2-\frac{3}{4} \zeta(3)=0.469941487 \ldots, \\
\mathscr{C}_{3}= & \frac{83}{72} \pi^{2} \zeta(3)-\frac{215}{24} \zeta(5)+\frac{100}{3}\left[\left(\operatorname{Li}_{4}\left(\frac{1}{2}\right)+\frac{\ln ^{4} 2}{24}\right)-\frac{\pi^{2} \ln ^{2} 2}{24}\right]-\frac{239}{2160} \pi^{4}+\frac{139}{18} \zeta(3) \\
& -\frac{298}{9} \pi^{2} \ln 2+\frac{17101}{810} \pi^{2}+\frac{28259}{5184}=1.181241456 \ldots, \\
\mathscr{A}_{3}= & -\frac{17}{24} \pi^{2} \zeta(3)+\frac{25}{8} \zeta(5)-\frac{217}{9}\left(\operatorname{Li}_{4}\left(\frac{1}{2}\right)+\frac{\ln ^{4} 2}{24}\right)-\frac{103}{1080} \pi^{2} \ln ^{2} 2+\frac{3899}{25920} \pi^{4}-\frac{2929}{288} \zeta(3) \\
& +\frac{41671}{2160} \pi^{2} \ln 2-\frac{454979}{38880} \pi^{2}-\frac{77513}{186624}=0.171720018 \ldots
\end{aligned}
$$

In the above expression $\zeta(n)=\sum_{i=1}^{\infty} i^{-n}$.

\subsection{Four-loop contributions}

The four-loop coefficients $\mathscr{C}_{4}$ and $\mathscr{A}_{4}$ were obtained recently [9,10]; the expressions are much more cumbersome:

$$
\begin{aligned}
\mathscr{C}_{4}= & \frac{1243127611}{130636800}+\frac{30180451}{25920} \zeta(2)-\frac{255842141}{2721600} \zeta(3)-\frac{8873}{3} \zeta(2) \ln 2+\frac{6768227}{2160} \zeta(4)+\frac{19063}{360} \zeta(2) \ln ^{2} 2 \\
& +\frac{12097}{90}\left(a_{4}+\frac{1}{24} \ln ^{4} 2\right)-\frac{2862857}{6480} \zeta(5)-\frac{12720907}{64800} \zeta(3) \zeta(2)-\frac{221581}{2160} \zeta(4) \ln 2+\frac{9656}{27}\left(a_{5}+\frac{1}{12} \zeta(2) \ln ^{3} 2\right. \\
& \left.-\frac{1}{120} \ln ^{5} 2\right)+\frac{191490607}{46656} \zeta(6)+\frac{10358551}{43200} \zeta^{2}(3)-\frac{40136}{27} a_{6}+\frac{26404}{27} b_{6}-\frac{700706}{675} a_{4} \zeta(2)-\frac{26404}{27} a_{5} \ln 2 \\
& +\frac{26404}{27} \zeta(5) \ln 2-\frac{63749}{50} \zeta(3) \zeta(2) \ln 2-\frac{40723}{135} \zeta(4) \ln ^{2} 2+\frac{13202}{81} \zeta(3) \ln ^{3} 2-\frac{253201}{2700} \zeta(2) \ln ^{4} 2+\frac{7657}{1620} \ln ^{6} 2 \\
& +\frac{2895304273}{435456} \zeta(7)+\frac{670276309}{193536} \zeta(4) \zeta(3)+\frac{85933}{63} a_{4} \zeta(3)+\frac{7121162687}{967680} \zeta(5) \zeta(2)-\frac{142793}{18} a_{5} \zeta(2)-\frac{195848}{21} a_{7} \\
& +\frac{195848}{63} b_{7}-\frac{116506}{189} d_{7}-\frac{4136495}{384} \zeta(6) \ln 2-\frac{1053568}{189} a_{6} \ln 2+\frac{233012}{189} b_{6} \ln 2+\frac{407771}{432} \zeta^{2}(3) \ln 2
\end{aligned}
$$


$-\frac{8937}{2} a_{4} \zeta(2) \ln 2+\frac{833683}{3024} \zeta(5) \ln ^{2} 2-\frac{3995099}{6048} \zeta(3) \zeta(2) \ln ^{2} 2-\frac{233012}{189} a_{5} \ln ^{2} 2+\frac{1705273}{1512} \zeta(4) \ln ^{3} 2$

$+\frac{602303}{4536} \zeta(3) \ln ^{4} 2-\frac{1650461}{11340} \zeta(2) \ln ^{5} 2+\frac{52177}{15876} \ln ^{7} 2+\sqrt{3}\left[-\frac{14101}{480} \mathrm{Cl}_{4}\left(\frac{\pi}{3}\right)-\frac{169703}{1440} \zeta(2) \mathrm{Cl}_{2}\left(\frac{\pi}{3}\right)\right.$

$+\frac{494}{27} \operatorname{Im} H_{0,0,0,1,-1,-1}\left(e^{i \frac{\pi}{3}}\right)+\frac{494}{27} \operatorname{Im} H_{0,0,0,1,-1,1}\left(e^{i \frac{2 \pi}{3}}\right)+\frac{494}{27} \operatorname{Im} H_{0,0,0,1,1,-1}\left(e^{i \frac{2 \pi}{3}}\right)+19 \operatorname{Im} H_{0,0,1,0,1,1}\left(e^{i \frac{2 \pi}{3}}\right)$

$+\frac{437}{12} \operatorname{Im} H_{0,0,0,1,1,1}\left(e^{i \frac{2 \pi}{3}}\right)+\frac{29812}{297} \mathrm{Cl}_{6}\left(\frac{\pi}{3}\right)+\frac{4940}{81} a_{4} \mathrm{Cl}_{2}\left(\frac{\pi}{3}\right)-\frac{520847}{69984} \zeta(5) \pi-\frac{129251}{81} \zeta(4) \mathrm{Cl}_{2}\left(\frac{\pi}{3}\right)$

$-\frac{892}{15} \operatorname{Im} H_{0,1,1,-1}\left(e^{i \frac{2 \pi}{3}}\right) \zeta(2)-\frac{1784}{45} \operatorname{Im} H_{0,1,1,-1}\left(e^{i \frac{\pi}{3}}\right) \zeta(2)+\frac{1729}{54} \zeta(3) \operatorname{Im} H_{0,1,-1}\left(e^{i \frac{\pi}{3}}\right)$

$+\frac{1729}{36} \zeta(3) \operatorname{Im} H_{0,1,1}\left(e^{i \frac{2 \pi}{3}}\right)+\frac{837190}{729} \mathrm{Cl}_{4}\left(\frac{\pi}{3}\right) \zeta(2)+\frac{25937}{4860} \zeta(3) \zeta(2) \pi-\frac{223}{243} \zeta(4) \pi \ln 2$

$\left.+\frac{892}{9} \operatorname{Im} H_{0,1,-1}\left(e^{i \frac{\pi}{3}}\right) \zeta(2) \ln 2+\frac{446}{3} \operatorname{Im} H_{0,1,1}\left(e^{i \frac{2 \pi}{3}}\right) \zeta(2) \ln 2-\frac{7925}{81} \mathrm{Cl}_{2}\left(\frac{\pi}{3}\right) \zeta(2) \ln ^{2} 2+\frac{1235}{486} \mathrm{Cl}_{2}\left(\frac{\pi}{3}\right) \ln ^{4} 2\right]$

$+\frac{13487}{60}\left(\operatorname{Re}_{0,0,0,1,0,1}\left(e^{i \frac{\pi}{3}}\right)+\mathrm{Cl}_{4}\left(\frac{\pi}{3}\right) \mathrm{Cl}_{2}\left(\frac{\pi}{3}\right)\right)+\frac{136781}{360} \mathrm{Cl}_{2}^{2}\left(\frac{\pi}{3}\right) \zeta(2)+\frac{651}{4} \operatorname{Re}_{0,0,0,1,0,1,-1}\left(e^{i \frac{\pi}{3}}\right)$

$+651 \operatorname{Re} H_{0,0,0,0,1,1,-1}\left(e^{i \frac{\pi}{3}}\right)-\frac{17577}{32} \operatorname{Re} H_{0,0,1,0,0,1,1}\left(e^{i \frac{2 \pi}{3}}\right)-\frac{87885}{64} \operatorname{Re} H_{0,0,0,1,0,1,1}\left(e^{i \frac{2 \pi}{3}}\right)$

$-\frac{17577}{8} \operatorname{Re} H_{0,0,0,0,1,1,1}\left(e^{i \frac{2 \pi}{3}}\right)+\frac{651}{4} \mathrm{Cl}_{4}\left(\frac{\pi}{3}\right) \operatorname{Im} H_{0,1,-1}\left(e^{i \frac{\pi}{3}}\right)+\frac{1953}{8} \mathrm{Cl}_{4}\left(\frac{\pi}{3}\right) \operatorname{Im} H_{0,1,1}\left(e^{i \frac{2 \pi}{3}}\right)$

$+\frac{31465}{176} \mathrm{Cl}_{6}\left(\frac{\pi}{3}\right) \pi+\frac{211}{4} \operatorname{Re} H_{0,1,0,1,-1}\left(e^{i \frac{\pi}{3}}\right) \zeta(2)+\frac{211}{2} \operatorname{Re} H_{0,0,1,1,-1}\left(e^{i \frac{\pi}{3}}\right) \zeta(2)+\frac{1899}{16} \operatorname{Re} H_{0,1,0,1,1}\left(e^{i \frac{2 \pi}{3}}\right) \zeta(2)$

$+\frac{1899}{8} \operatorname{Re} H_{0,0,1,1,1}\left(e^{i \frac{2 \pi}{3}}\right) \zeta(2)+\frac{211}{4} \operatorname{Im} H_{0,1,-1}\left(e^{i \frac{\pi}{3}}\right) \mathrm{Cl}_{2}\left(\frac{\pi}{3}\right) \zeta(2)+\frac{633}{8} \operatorname{Im} H_{0,1,1}\left(e^{i \frac{2 \pi}{3}}\right) \mathrm{Cl}_{2}\left(\frac{\pi}{3}\right) \zeta(2)$

$-\frac{28276}{25} \zeta(2) \mathrm{Cl}_{2}\left(\frac{\pi}{2}\right)^{2}+104\left(4 \operatorname{Re} H_{0,1,0,1,1}(i) \zeta(2)+4 \operatorname{Im} H_{0,1,1}(i) \mathrm{Cl}_{2}\left(\frac{\pi}{2}\right) \zeta(2)-2 \mathrm{Cl}_{4}\left(\frac{\pi}{2}\right) \zeta(2) \pi\right.$

$\left.+\mathrm{Cl}_{2}^{2}\left(\frac{\pi}{2}\right) \zeta(2) \ln 2\right)+\sqrt{3}\left[\pi\left(-\frac{28458503}{691200} B_{3}+\frac{250077961}{18662400} C_{3}\right)+\frac{483913}{77760} \pi f_{2}(0,0,1)+\pi\left(\frac{4715}{1944} \ln 2 f_{2}(0,0,1)\right.\right.$

$\left.+\frac{270433}{10935} f_{2}(0,2,0)-\frac{188147}{4860} f_{2}(0,1,1)+\frac{188147}{12960} f_{2}(0,0,2)\right)+\pi\left(\frac{826595}{248832} \zeta(2) f_{2}(0,0,1)-\frac{5525}{432} \ln 2 f_{2}(0,0,2)\right.$

$+\frac{5525}{162} \ln 2 f_{2}(0,1,1)-\frac{5525}{243} \ln 2 f_{2}(0,2,0)+\frac{526015}{248832} f_{2}(0,0,3)-\frac{4675}{768} f_{2}(0,1,2)+\frac{1805965}{248832} f_{2}(0,2,1)$

$-\frac{3710675}{1119744} f_{2}(0,3,0)-\frac{75145}{124416} f_{2}(1,0,2)-\frac{213635}{124416} f_{2}(1,1,1)+\frac{168455}{62208} f_{2}(1,2,0)+\frac{75145}{248832} f_{2}(2,0,1)$

$\left.\left.+\frac{69245}{124416} f_{2}(2,1,0)\right)\right]-\frac{4715}{1458} \zeta(2) f_{1}(0,0,1)+\zeta(2)\left(\frac{2541575}{82944} f_{1}(0,0,2)-\frac{556445}{6912} f_{1}(0,1,1)+\frac{54515}{972} f_{1}(0,2,0)\right.$

$\left.-\frac{75145}{20736} f_{1}(1,0,1)\right)-\frac{541}{300} C_{81 a}-\frac{629}{60} C_{81 b}+\frac{49}{3} C_{81 c}-\frac{327}{160} C_{83 a}+\frac{49}{36} C_{83 b}+\frac{37}{6} C_{83 c}=-1.912245764 \ldots$,

$$
\begin{aligned}
& \mathscr{A}_{4}=-\frac{92473962293}{19752284160}-\frac{6619898477}{21772800} \zeta(2)-\frac{12334741}{132300} \zeta(3)+\frac{97832509}{90720} \zeta(2) \ln 2-\frac{241619904061}{391910400} \zeta(4) \\
& +\frac{4572662443}{12247200} \ln ^{2} 2 \zeta(2)-\frac{1449791143}{3061800}\left(a_{4}+\frac{1}{24} \ln ^{4} 2\right)+\frac{90355973}{134400} \zeta(5)+\frac{1173056009}{9072000} \zeta(3) \zeta(2) \\
& -\frac{8548241}{30240} \zeta(4) \ln 2-\frac{68168}{135}\left(a_{5}+\frac{1}{12} \zeta(2) \ln ^{3} 2-\frac{1}{120} \ln ^{5} 2\right)-\frac{244603373713}{52254720} \zeta(6)-\frac{8082848863}{24192000} \zeta^{2}(3) \\
& +\frac{26062}{27} a_{6}-\frac{18215}{27} b_{6}+\frac{18215}{27} a_{5} \ln 2-\frac{18215}{27} \zeta(5) \ln 2+\frac{402152509}{189000} a_{4} \zeta(2)+\frac{159693503}{72000} \zeta(3) \zeta(2) \ln 2 \\
& -\frac{328317209}{302400} \zeta(4) \ln ^{2} 2-\frac{18215}{162} \zeta(3) \ln ^{3} 2+\frac{188648503}{1512000} \zeta(2) \ln ^{4} 2-\frac{21671}{6480} \ln ^{6} 2-\frac{7224951103}{1741824} \zeta(7) \\
& -\frac{1267114025}{387072} \zeta(4) \zeta(3)-\frac{427145}{504} a_{4} \zeta(3)-\frac{2749470791}{387072} \zeta(5) \zeta(2)+\frac{1420289}{180} a_{5} \zeta(2)+\frac{116987}{21} a_{7}-\frac{116987}{63} b_{7} \\
& +\frac{256321}{756} d_{7}+\frac{971827}{128} \zeta(6) \ln 2+\frac{607282}{189} a_{6} \ln 2-\frac{256321}{378} b_{6} \ln 2-\frac{1794247}{3456} \zeta^{2}(3) \ln 2+\frac{104041}{20} a_{4} \zeta(2) \ln 2 \\
& -\frac{1888991}{24192} \zeta(5) \ln ^{2} 2+\frac{75222353}{60480} \zeta(3) \zeta(2) \ln ^{2} 2+\frac{256321}{378} a_{5} \ln ^{2} 2-\frac{9699379}{6048} \zeta(4) \ln ^{3} 2-\frac{2574883}{36288} \zeta(3) \ln ^{4} 2
\end{aligned}
$$




$$
\begin{aligned}
& +\frac{37144753}{226800} \zeta(2) \ln ^{5} 2-\frac{218465}{127008} \ln ^{7} 2+\sqrt{3}\left[-\frac{14186171}{194400} \mathrm{Cl}_{4}\left(\frac{\pi}{3}\right)-\frac{103023803}{583200} \zeta(2) \mathrm{Cl}_{2}\left(\frac{\pi}{3}\right)\right. \\
& +\frac{916598}{76545} \operatorname{Im} H_{0,0,0,1,-1,-1}\left(e^{i \frac{\pi}{3}}\right)+\frac{916598}{76545} \operatorname{Im} H_{0,0,0,1,-1,1}\left(e^{i \frac{2 \pi}{3}}\right)+\frac{916598}{76545} \operatorname{Im} H_{0,0,0,1,1,-1}\left(e^{i \frac{2 \pi}{3}}\right) \\
& +\frac{458299}{36855} \operatorname{Im} H_{0,0,1,0,1,1}\left(e^{i \frac{2 \pi}{3}}\right)+\frac{10540877}{442260} \operatorname{Im} H_{0,0,0,1,1,1}\left(e^{i \frac{2 \pi}{3}}\right)+\frac{178619489}{3980340} \mathrm{Cl}_{6}\left(\frac{\pi}{3}\right)+\frac{1833196}{45927} a_{4} \mathrm{Cl}_{2}\left(\frac{\pi}{3}\right) \\
& -\frac{12563350487}{2579260320} \zeta(5) \pi+\frac{533401067}{459270} \zeta(4) \mathrm{Cl}_{2}\left(\frac{\pi}{3}\right)+\frac{844343}{18900} \operatorname{Im} H_{0,1,1,-1}\left(e^{i \frac{2 \pi}{3}}\right) \zeta(2) \\
& +\frac{844343}{28350} \operatorname{Im} H_{0,1,1,-1}\left(e^{i \frac{\pi}{3}}\right) \zeta(2)+\frac{458299}{21870} \zeta(3) \operatorname{Im} H_{0,1,-1}\left(e^{i \frac{\pi}{3}}\right)+\frac{458299}{14580} \zeta(3) \operatorname{Im} H_{0,1,1}\left(e^{i \frac{2 \pi}{3}}\right) \\
& -\frac{263673944}{295245} \mathrm{Cl}_{4}\left(\frac{\pi}{3}\right) \zeta(2)-\frac{39924629}{6889050} \zeta(3) \zeta(2) \pi+\frac{844343}{1224720} \zeta(4) \pi \ln 2-\frac{844343}{11340} \operatorname{Im} H_{0,1,-1}\left(e^{i \frac{\pi}{3}}\right) \zeta(2) \ln 2 \\
& \left.-\frac{844343}{7560} \operatorname{Im} H_{0,1,1}\left(e^{i \frac{2 \pi}{3}}\right) \zeta(2) \ln 2+\frac{458299}{275562} \mathrm{Cl}_{2}\left(\frac{\pi}{3}\right) \ln ^{4} 2+\frac{19130869}{367416} \zeta(2) \mathrm{Cl}_{2}\left(\frac{\pi}{3}\right) \ln ^{2} 2\right] \\
& +\frac{212671}{2400}\left(\operatorname{Re} H_{0,0,0,1,0,1}\left(e^{i \frac{\pi}{3}}\right)+\mathrm{Cl}_{4}\left(\frac{\pi}{3}\right) \mathrm{Cl}_{2}\left(\frac{\pi}{3}\right)\right)-\frac{1031987}{14400} \mathrm{Cl}_{2}^{2}\left(\frac{\pi}{3}\right) \zeta(2)-\frac{507}{4} \operatorname{Re}_{0,0,0,1,0,1,-1}\left(e^{i \frac{\pi}{3}}\right) \\
& -507 \operatorname{Re} H_{0,0,0,0,1,1,-1}\left(e^{i \frac{\pi}{3}}\right)+\frac{13689}{32} \operatorname{Re} H_{0,0,1,0,0,1,1}\left(e^{i \frac{2 \pi}{3}}\right)+\frac{68445}{64} \operatorname{Re} H_{0,0,0,1,0,1,1}\left(e^{i \frac{2 \pi}{3}}\right) \\
& +\frac{13689}{8} \operatorname{Re} H_{0,0,0,0,1,1,1}\left(e^{i \frac{2 \pi}{3}}\right)-\frac{507}{4} \mathrm{Cl}_{4}\left(\frac{\pi}{3}\right) \operatorname{Im} H_{0,1,-1}\left(e^{i \frac{\pi}{3}}\right)-\frac{1521}{8} \mathrm{Cl}_{4}\left(\frac{\pi}{3}\right) \operatorname{Im} H_{0,1,1}\left(e^{i \frac{2 \pi}{3}}\right) \\
& -\frac{24505}{176} \mathrm{Cl}_{6}\left(\frac{\pi}{3}\right) \pi-\frac{295}{4} \operatorname{Re} H_{0,1,0,1,-1}\left(e^{i \frac{\pi}{3}}\right) \zeta(2)-\frac{295}{2} \operatorname{Re} H_{0,0,1,1,-1}\left(e^{i \frac{\pi}{3}}\right) \zeta(2)-\frac{2655}{16} \operatorname{Re} H_{0,1,0,1,1}\left(e^{i \frac{2 \pi}{3}}\right) \zeta(2) \\
& -\frac{2655}{8} \operatorname{Re} H_{0,0,1,1,1}\left(e^{i \frac{2 \pi}{3}}\right) \zeta(2)-\frac{295}{4} \operatorname{Im} H_{0,1,-1}\left(e^{i \frac{\pi}{3}}\right) \mathrm{Cl}_{2}\left(\frac{\pi}{3}\right) \zeta(2)-\frac{885}{8} \operatorname{Im} H_{0,1,1}\left(e^{i \frac{2 \pi}{3}}\right) \mathrm{Cl}_{2}\left(\frac{\pi}{3}\right) \zeta(2) \\
& -\frac{1117}{36} \zeta(2) \mathrm{Cl}_{2}\left(\frac{\pi}{2}\right)+\frac{38424}{125} \zeta(2) \mathrm{Cl}_{2}^{2}\left(\frac{\pi}{2}\right)-118\left(4 \operatorname{Re} H_{0,1,0,1,1}(i) \zeta(2)+4 \operatorname{Im} H_{0,1,1}(i) \mathrm{Cl}_{2}\left(\frac{\pi}{2}\right) \zeta(2)\right. \\
& \left.-2 \mathrm{Cl}_{4}\left(\frac{\pi}{2}\right) \zeta(2) \pi+\mathrm{Cl}_{2}^{2}\left(\frac{\pi}{2}\right) \zeta(2) \ln 2\right)+\sqrt{3}\left[\pi\left(+\frac{5581729229}{362880000} B_{3}+\frac{1233637481}{1399680000} C_{3}\right)-\frac{11495611}{3265920} \pi f_{2}(0,0,1)\right. \\
& +\pi\left(\frac{751}{972} \ln 2 f_{2}(0,0,1)-\frac{365478661}{24494400} f_{2}(0,2,0)+\frac{119022487}{5443200} f_{2}(0,1,1)-\frac{119022487}{14515200} f_{2}(0,0,2)\right) \\
& -\frac{751}{729} \zeta(2) f_{1}(0,0,1)+\pi\left(-\frac{1735283}{497664} \zeta(2) f_{2}(0,0,1)+\frac{1105}{108} \ln 2 f_{2}(0,0,2)-\frac{2210}{81} \ln 2 f_{2}(0,1,1)\right. \\
& +\frac{4420}{243} \ln 2 f_{2}(0,2,0)-\frac{1104271}{497664} f_{2}(0,0,3)+\frac{272833}{41472} f_{2}(0,1,2)-\frac{4011005}{497664} f_{2}(0,2,1)+\frac{8417635}{2239488} f_{2}(0,3,0) \\
& \left.\left.+\frac{157753}{248832} f_{2}(1,0,2)+\frac{354323}{248832} f_{2}(1,1,1)-\frac{298711}{124416} f_{2}(1,2,0)-\frac{157753}{497664} f_{2}(2,0,1)-\frac{98285}{248832} f_{2}(2,1,0)\right)\right] \\
& +\zeta(2)\left(-\frac{4629335}{165888} f_{1}(0,0,2)+\frac{112357}{1536} f_{1}(0,1,1)-\frac{99731}{1944} f_{1}(0,2,0)+\frac{157753}{41472} f_{1}(1,0,1)\right) \\
& +\frac{174623}{288000} C_{81 a}+\frac{29479}{7200} C_{81 b}-\frac{43}{6} C_{81 c}+\frac{10871}{14400} C_{83 a}-\frac{157}{1620} C_{83 b}-\frac{95}{24} C_{83 c}=0.886545673 \ldots
\end{aligned}
$$

In the above expressions $a_{n}=\sum_{i=1}^{\infty} 2^{-i} i^{-n}, b_{6}=H_{0,0,0,0,1,1}\left(\frac{1}{2}\right), b_{7}=H_{0,0,0,0,0,1,1}\left(\frac{1}{2}\right)$, $d_{7}=H_{0,0,0,0,1,-1,-1}(1), \mathrm{Cl}_{n}(\theta)=\operatorname{Im~Li}_{n}\left(e^{i \theta}\right) . H_{i_{1}, i_{2}, \ldots}(x)$ are the harmonic polylogarithms [11].

The integrals $f_{j}$ are defined as follows:

$$
\begin{aligned}
f_{m}(i, j, k) & =\int_{1}^{9} d s D_{1}(s) \operatorname{Re}\left(\sqrt{3^{m-1}} D_{m}(s)\right)\left(s-\frac{9}{5}\right) \ln ^{i}(9-s) \ln ^{j}(s-1) \ln ^{k}(s) \\
D_{m}(s) & =\frac{2}{\sqrt{(\sqrt{s}+3)(\sqrt{s}-1)^{3}}} K\left(m-1-(2 m-3) \frac{(\sqrt{s}-3)(\sqrt{s}+1)^{3}}{(\sqrt{s}+3)(\sqrt{s}-1)^{3}}\right) .
\end{aligned}
$$

$C_{8 x y}$ are constants known only numerically, being related to a double elliptic kernel of weight six. 
We see that the expression contain a polylogarithmic part and an elliptic part. The polylogarithmic part contains $\zeta(n), a_{n}, b_{m}, d_{7}$ and $H_{\{m\}}\left(e^{i x}\right)$; the elliptic part contains $B_{3}, C_{3}$ (highlighted in red) and $f_{m}(i, j, k)$; all these objects are defined as one-dimensional integrals of products of elliptic integrals. In particular $B_{3}$ and $C_{3}$ have the lowest weight (3) and therefore are the first choice for a more detailed analysis, which will be discussed in the next section.

\section{Four "elliptic" constants}

We define the four constants $A_{3}, B_{3}, C_{3}, D_{3}$ with the following integral representations [12]:

$$
\begin{array}{ll}
A_{3}=\int_{0}^{1} d x \frac{K_{c}(x) K_{c}(1-x)}{\sqrt{1-x}}=4.860756383 \ldots, & B_{3}=\int_{0}^{1} d x \frac{K_{c}^{2}(x)}{\sqrt{1-x}}=7.396099534 \ldots, \\
C_{3}=\int_{0}^{1} d x \frac{E_{c}^{2}(x)}{\sqrt{1-x}}=2.376887326 \ldots, & D_{3}=\int_{0}^{1} d x \frac{E_{c}(x) E_{c}(1-x)}{\sqrt{1-x}}=2.506147295 \ldots, \\
K_{c}(x)=\frac{2 \pi}{\sqrt{27}} 2 F_{1}\left(\begin{array}{c}
\frac{1}{3} \frac{2}{3} ; x \\
1
\end{array}\right), & E_{c}(x)=\frac{2 \pi}{\sqrt{27}} 2 F_{1}\left(\begin{array}{c}
\left.\frac{1}{3}-\frac{1}{3} ; x\right) . \\
1
\end{array}\right)
\end{array}
$$

$B_{3}$ and $C_{3}$ appeared for the first time in the expression of the scalar master integral of the 4-loop on-shell massive sunrise diagram $S_{4}(D)$ in $D$ dimensions [12]

$$
\begin{aligned}
S_{4}(D=4-2 \varepsilon) & =-\frac{5}{2 \varepsilon^{4}}-\frac{45}{4 \varepsilon^{3}}-\frac{4255}{144 \varepsilon^{2}}-\frac{106147}{1728 \varepsilon}+\frac{\pi \sqrt{3}}{240}\left(297 B_{3}-1477 C_{3}\right)-\frac{2320981}{20736}+O(\varepsilon), \\
S_{4}(D=2) & =\sqrt{3} \pi B_{3} .
\end{aligned}
$$

This integral is one of the master integrals needed to calculate the 4-loop contributions $\mathscr{C}_{4}$ and $\mathscr{A}_{4}$. The constant $A_{3}$ appears also in intermediate results of four-loop calculations of Ref. [9, 10], and cancels out in the final result.

\subsection{Hypergeometric expressions}

If we define the regularized hypergeometric ${ }_{4} \tilde{F}_{3}$

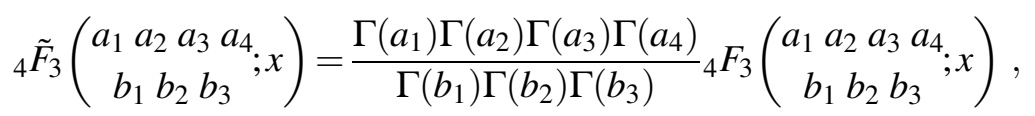

we can obtain hypergeometric expressions for these constants $[9,10,13]$ :

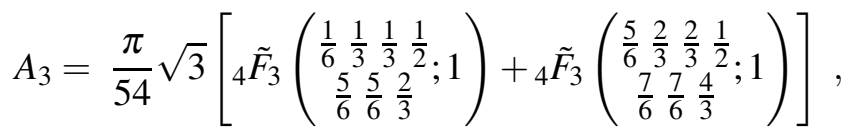

$$
\begin{aligned}
& B_{3}=\frac{\pi}{27} \sqrt{3}\left[{ }_{4} \tilde{F}_{3}\left(\begin{array}{cccc}
\frac{1}{6} & \frac{1}{3} & \frac{1}{3} & \frac{1}{2} \\
\frac{5}{6} & \frac{5}{6} & \frac{2}{3}
\end{array}\right)-{ }_{4} \tilde{F}_{3}\left(\begin{array}{cccc}
\frac{5}{6} & \frac{2}{3} & \frac{2}{3} & \frac{1}{2} \\
\frac{7}{6} & \frac{7}{6} & \frac{4}{3}
\end{array}\right)\right] \text {, }
\end{aligned}
$$




$$
\begin{aligned}
& C_{3}=\frac{\pi}{27} \sqrt{3}\left[{ }_{4} \tilde{F}_{3}\left(\begin{array}{c}
\frac{1}{6} \frac{1}{3} \frac{4}{3}-\frac{1}{2} ; 1 \\
-\frac{1}{6} \frac{5}{6} \frac{5}{3}
\end{array}\right)-{ }_{4} \tilde{F}_{3}\left(\begin{array}{c}
-\frac{7}{6}-\frac{1}{3} \frac{2}{3}-\frac{1}{2} ; 1 \\
-\frac{5}{6} \frac{1}{6} \frac{1}{3}
\end{array}\right)\right], \\
& D_{3}=\frac{-\pi}{180} \sqrt{3}\left[{ }_{4} \tilde{F}_{3}\left(\begin{array}{c}
\frac{1}{6} \frac{1}{3} \frac{4}{3}-\frac{1}{2} ; 1 \\
-\frac{1}{6} \frac{5}{6} \frac{5}{3}
\end{array}\right)+{ }_{4} \tilde{F}_{3}\left(\begin{array}{c}
-\frac{7}{6}-\frac{1}{3} \frac{2}{3}-\frac{1}{2} ; 1 \\
-\frac{5}{6} \frac{1}{6} \frac{1}{3}
\end{array}\right)\right]+\frac{3}{10} A_{3}+\frac{2}{9} \pi \sqrt{3} .
\end{aligned}
$$

Note that in the above expressions the sum and the difference of the same hypergeometric values appear.

\subsection{Bessel Moments}

The constants Eqs. (2.1)-(2.2) are related to the Bessel moments [14,15]

$$
M(a, b, c)=\int_{0}^{\infty} I_{0}^{a}(x) K_{0}^{b}(x) x^{c} d x
$$

$I_{0}(x), K_{0}(x)$ are the modified Bessel functions. The following four moments can be written in terms of the constants $A_{3}, B_{3}, C_{3}, D_{3}$ :

$$
\begin{aligned}
& M(1,5,1)=\frac{\sqrt{3}}{16} \pi B_{3}=\frac{1}{16} S_{4}(2), \\
& M(1,5,3)=\frac{\sqrt{3}}{384} \pi\left(3 B_{3}-7 C_{3}\right), \\
& M(2,4,1)=\frac{9}{40} A_{3}, \\
& M(2,4,3)=\frac{1}{1440}\left(-54 A_{3}+315 D_{3}-70 \pi \sqrt{3}\right) .
\end{aligned}
$$

The following quadratic relation was conjectured [15] and proven [17, 18]:

$$
\operatorname{Det}\left[\begin{array}{l}
M(1,5,1) M(1,5,3) \\
M(2,4,1) M(2,4,3)
\end{array}\right]=\frac{\pi^{4}}{24^{2}}
$$

Substituting Eqs. (2.12)-(2.15) and Eqs. (2.7)-(2.10) in Eq. (2.16) one obtains:

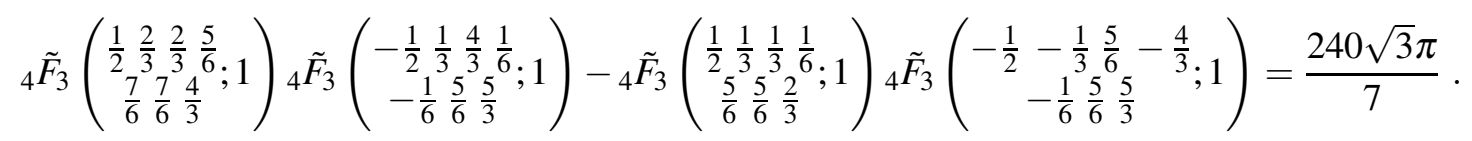

Note that in Ref. [16] Eq. (2.17) is written in terms of the plain ${ }_{4} F_{3}$ hypergeometric:

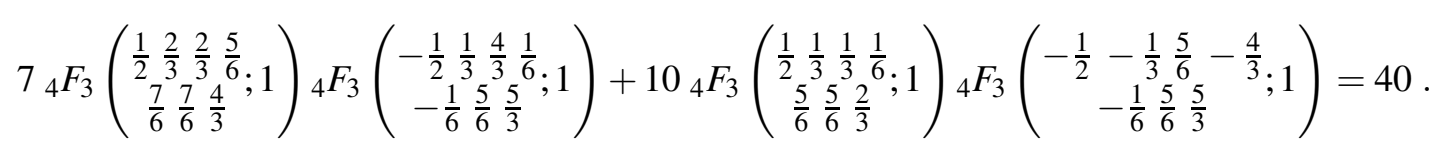

For more information on quadratic relations, see for example Refs. [18-20]. 


\subsection{Plain elliptic case}

Let us consider constants defined similarly to Eqs. (2.1)-(2.2) with the plain elliptic integrals instead of the "cubic" version, and a factor $\frac{1}{2}$ for a better match to the definitions in the literature.

$$
\begin{aligned}
& \bar{A}_{3}=\int_{0}^{1} d x \frac{K(x) K(1-x)}{2 \sqrt{1-x}}=4.335593665 \ldots, \quad \bar{B}_{3}=\int_{0}^{1} d x \frac{K^{2}(x)}{2 \sqrt{1-x}}=6.997563016 \ldots \\
& \bar{C}_{3}=\int_{0}^{1} d x \frac{E^{2}(x)}{2 \sqrt{1-x}}=1.552273101 \ldots \\
& \bar{D}_{3}=\int_{0}^{1} d x \frac{E(x) E(1-x)}{2 \sqrt{1-x}}=1.721167544 \ldots \\
& K(x)=\frac{\pi}{2}{ }_{2} F_{1}\left(\begin{array}{c}
\frac{1}{2} \frac{1}{2} \\
1
\end{array} ; x\right) \\
& E(x)=\frac{\pi}{2}{ }_{2} F_{1}\left(\begin{array}{c}
\frac{1}{2}-\frac{1}{2} ; x \\
1
\end{array}\right) \\
& \bar{A}_{3}=\frac{\pi}{8}{ }_{4} \tilde{F}_{3}\left(\begin{array}{cccc}
\frac{1}{2} & \frac{1}{2} & \frac{1}{2} & \frac{1}{2} \\
1 & 1 & 1
\end{array} ; 1\right)
\end{aligned}
$$

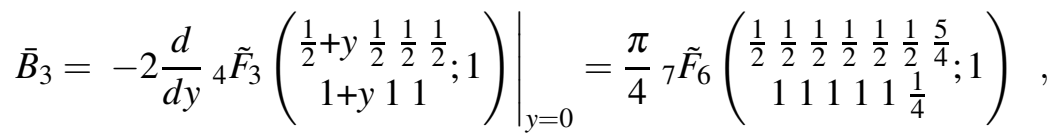

$$
\begin{aligned}
& \bar{C}_{3}=\frac{1}{3} \bar{B}_{3}+\left.\frac{1}{48} \frac{d}{d y}{ }_{4} \tilde{F}_{3}\left(\begin{array}{c}
-\frac{1}{2}+y-\frac{1}{2} \frac{1}{2}+y \frac{1}{2} ; 1 \\
1+y 1+y 1
\end{array}\right)\right|_{y=0}, \\
& \bar{D}_{3}=-\frac{\pi}{48}{ }_{4} \tilde{F}_{3}\left(\begin{array}{cccc}
\frac{1}{2} & \frac{1}{2} & \frac{1}{2} & -\frac{1}{2} \\
1 & 1 & 1
\end{array}\right)+\frac{\pi}{6},
\end{aligned}
$$

I we compare Eqs. (2.7)-(2.10) with Eqs. (2.22)-(2.25), we can see Eqs. (2.22)-(2.25) as a singular case where the two hypergeometric terms have the same indices; therefore the differences in Eqs. (2.8)-(2.9) correspond to derivatives w.r.t. the indices in Eqs. (2.23)-(2.24), and the sums become single terms. The corresponding Bessel moments

$$
\begin{aligned}
& M(0,4,0)=2 \pi \bar{A}_{3}, \\
& M(0,4,2)=\frac{\pi}{2} \bar{A}_{3}-\frac{9 \pi}{4} \bar{D}_{3}+\frac{9}{16} \pi^{2}, \\
& M(1,3,0)=\bar{B}_{3}, \\
& M(1,3,2)=-\frac{1}{32} \bar{B}_{3}+\frac{9}{32} \bar{C}_{3},
\end{aligned}
$$

The determinant gives a quadratic relation:

$$
\operatorname{Det}\left[\begin{array}{l}
M(0,4,0) M(0,4,2) \\
M(1,3,0) M(1,3,2)
\end{array}\right]=\frac{3}{64} \pi^{4} .
$$




\section{Conjectured quadratic relation}

Let's try to extend the quadratic relation 2.17.

As reference we consider a quadratic relation among ${ }_{2} F_{1}$ hypergeometric, the Elliott's identity [21]:

$$
\begin{aligned}
& +{ }_{2} F_{1}\left(\begin{array}{c}
\frac{1}{2}+\lambda-\frac{1}{2}-v \\
1+\lambda+\mu
\end{array} ; r\right){ }_{2} F_{1}\left(\begin{array}{c}
\frac{1}{2}-\lambda \frac{1}{2}+v \\
1+v+\mu
\end{array} ; 1-r\right)+{ }_{2} F_{1}\left(\begin{array}{c}
\frac{1}{2}+\lambda \frac{1}{2}-v \\
1+\lambda+\mu
\end{array} ; r\right){ }_{2} F_{1}\left(\begin{array}{c}
-\frac{1}{2}-\lambda \frac{1}{2}+v \\
1+v+\mu
\end{array} ; 1-r\right) \\
& -{ }_{2} F_{1}\left(\begin{array}{c}
\frac{1}{2}+\lambda \frac{1}{2}-v \\
1+\lambda+\mu
\end{array} ; r\right){ }_{2} F_{1}\left(\begin{array}{c}
\frac{1}{2}-\lambda \frac{1}{2}+v \\
1+v+\mu
\end{array} ; 1-r\right)=\frac{\Gamma(1+\lambda+\mu) \Gamma(1+v+\mu)}{\Gamma\left(\frac{3}{2}+\lambda+\mu+v\right) \Gamma\left(\frac{1}{2}+\mu\right)}
\end{aligned}
$$

for $\lambda=\mu=v=0$ this identity reduces to the Legendre's identity.

We assume that all the fractions appearing in the indices of Eq. (2.17) are actually $\frac{1}{2} \pm k \delta$ or $1 \pm k \delta$ with small integer $k$ and $\delta=\frac{1}{6}$,

In other words

$$
\begin{aligned}
\frac{1}{3} & \rightarrow \frac{1}{2}-\delta \\
\frac{2}{3} & \rightarrow \frac{1}{2}+\delta \\
\frac{5}{6} & \rightarrow \frac{1}{2}+2 \delta \\
-\frac{4}{3} & \rightarrow-\frac{3}{2}+\delta \text { or }-1-2 \delta, \ldots
\end{aligned}
$$

By verifying all possible combinations we found numerically that Eq. (2.17) corresponds to the case with $\delta=1 / 6$ of the general relation

$$
\begin{gathered}
+{ }_{4} \tilde{F}_{3}\left(\begin{array}{c}
\frac{1}{2} \frac{1}{2}+\delta \frac{1}{2}+\delta \frac{1}{2}+2 \delta \\
1+\delta 1+\delta 1+2 \delta
\end{array} ; 1\right){ }_{4} \tilde{F}_{3}\left(\begin{array}{c}
-\frac{1}{2} \frac{1}{2}-\delta \frac{3}{2}-\delta \frac{1}{2}-2 \delta ; 1 \\
-\delta 1-\delta 2-2 \delta
\end{array}\right) \\
-{ }_{4} \tilde{F}_{3}\left(\begin{array}{c}
\frac{1}{2} \frac{1}{2}-\delta \frac{1}{2}-\delta \frac{1}{2}-2 \delta \\
1-\delta 1-\delta 1-2 \delta
\end{array} ; 1\right){ }_{4} \tilde{F}_{3}\left(\begin{array}{c}
-\frac{1}{2}-\frac{1}{2}+\delta \frac{1}{2}+\delta-\frac{3}{2}+2 \delta ; 1 \\
-\delta 1-\delta 2-2 \delta
\end{array}\right)= \\
=\frac{4 \Gamma(1 / 2-2 \delta) \Gamma(1 / 2-\delta) \Gamma(-(1 / 2)+\delta) \Gamma(-(3 / 2)+2 \delta))}{\Gamma(1-\delta) \Gamma(-1+\delta)} \\
=128 \pi \frac{\delta-1}{(4 \delta-1)(2 \delta-3)(2 \delta-1)} \frac{\sin (\pi \delta)^{2}}{\sin (4 \pi \delta)^{2}}
\end{gathered}
$$

This conjecture can be rigorously proved likely by using intersection theory [22-24] along the same lines of similar identities for the simpler hypergeometric functions ${ }_{2} F_{1}$ and ${ }_{3} F_{2}$.

\section{References}

[1] J. Schwinger Phys. Rev. 73 (1948) 416. 
[2] J. Schwinger Phys. Rev. 75 (1949) 898.

[3] A. Petermann Helv. Phys. Acta 30 (1957) 407.

[4] C. M. Sommerfield Phys. Rev. 107 (1957) 328.

[5] S. Laporta and E.Remiddi Phys. Lett. B 379 (1996) 283.

[6] R. Barbieri, J. A. Mignaco and E. Remiddi, Nuovo Cim. A 11 (1972) 824.

[7] R. Barbieri, J. A. Mignaco and E. Remiddi, Nuovo Cim. A 11 (1972) 865.

[8] K. Melnikov and T. van Ritbergen, Phys. Rev. Lett. 84 (2000) 1673.

[9] S. Laporta, Phys. Lett. B 772 (2017) 232.

[10] S. Laporta, Phys. Lett. B 800 (2020), 135137

[11] E. Remiddi and J. A. M. Vermaseren, Int. J. Mod. Phys. A15 (2000) 725.

[12] S. Laporta, Int. J. Mod. Phys. A 23 (2008) 5007.

[13] Y. Zhou, arXiv:1801.02182 [math.CA].

[14] D. H. Bailey, J. M. Borwein, D. Broadhurst and M. L. Glasser, J. Phys. A 41 (2008), 205203 [arXiv:0801.0891 [hep-th]].

[15] D. Broadhurst and A. Mellit, PoS LL2016 (2016), 079

[16] D. Broadhurst and D. P. Roberts, PoS LL2018 (2018), 053

[17] Y. Zhou, Commun. Num. Theor. Phys. 12 (2018), 355-407

[18] Y. Zhou, arXiv:1801.05555 [math.NT].

[19] Y. Zhou, arXiv:2012.03523 [math.NT].

[20] J. Fresán, C. Sabbah and J. D. Yu, arXiv:2006.02702 [math.AG].

[21] E.B. Elliott, Messenger Math. 33 (1904) 31.

[22] P. Mastrolia and S. Mizera, JHEP 02 (2019), 139.

[23] H. Frellesvig, F. Gasparotto, S. Laporta, M. K. Mandal, P. Mastrolia, L. Mattiazzi and S. Mizera, JHEP 05 (2019), 153.

[24] H. Frellesvig, F. Gasparotto, M. K. Mandal, P. Mastrolia, L. Mattiazzi and S. Mizera, Phys. Rev. Lett. 123 (2019) no.20, 201602. 